\title{
Micro- and nano-fabricated implantable drug-delivery systems: current state and future perspectives
}

\author{
Advances in implantable drug-delivery pumps \\ promise to improve quality of life."
}

Keywords: drug delivery • implantable $\bullet$ microtechnology • nanotechology

\section{Biography}

Ellis Meng received her BS degree in engineering and applied science and her MS and $\mathrm{PhD}$ degrees in electrical engineering from the California Institute of Technology, Pasadena, in 1997, 1998 and 2003, respectively. She is a professor of Biomedical Engineering and Electrical Engineering at the University of Southern California, Los Angeles where she has been since 2004. She directs the Biomedical Microsystems Laboratory which specializes in biomicroelectromechanical systems, implantable biomedical microdevices, neural interfaces, and microfluidics. She is a recipient of the NSF CAREER, the Wallace H Coulter Foundation Early Career Translational Research, and the ASEE Curtis W McGraw Research Awards. In 2009, she was recognized as one of the TR35 Technology Review Young Innovators under 35.

Roya Sheybani received her BS (2008) and MS (2009) degrees in biomedical engineering from the University of Southern California, Los Angeles, where she is currently a $\mathrm{PhD}$ candidate in biomedical engineering. She is a member of Tau Beta Pi and was coauthor of an Outstanding Paper Award from the 15th International Conference on Solid-State Sensors, Actuators and Microsystems. She also received the best poster award at the 16th Annual Fred S Grodins Graduate Research Symposium (Grodins, 2012) and the 2nd place Wallace H Coulter Translational Research Partnership innovation award (2013). She is developing closed-loop implantable wireless microelectromechanical systems (MEMS) drug-delivery devices for management of chronic diseases.
Drug therapy plays a critical role in the treatment and management of many chronic conditions. Its efficacy is, in part, linked to the administration route and regimen. Many systemically administered drugs are associated with severe side effects [1] that dramatically impact quality of life. Also, while many novel pharmaceutical compounds, including biologics, gene therapies, small molecules and other nanoparticle-based therapeutics, have high specificity and potency, they possess limited bioactivity, relatively short halflife and stability, and have difficulty bypassing physiological barriers to reach targeted tissues [2]. These factors contribute to their limited compatibility with oral or parenteral routes of administration. These administration methods pose challenges for long-term treatment, are associated with a narrow therapeutic window, and require complex dosing schedules with combination therapy or labile active ingredients [3].

Implantable drug-delivery devices can target drug delivery to specific tissues, thereby minimizing side effects associated with systemic delivery. They also improve titration, provide automation and improve compliance. Completely implanted devices can reduce discomfort and eliminate infection risks from transcutaneous parts [4]. Folkman and Long pioneered implantable drugdelivery systems by introducing polymeric membranes (silicone rubber) for controlling release rates in the 1960s [5]. Since then, micro- and nano-fabrication technologies have enabled implantable miniaturized drugdelivery systems that can provide the desired drug release profile [4]. Drug administration

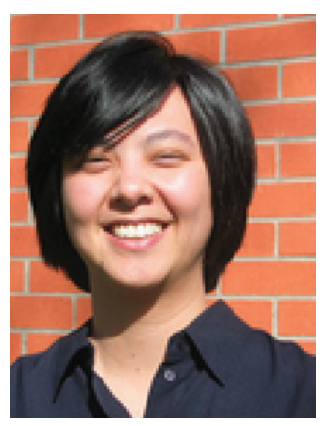

Ellis Meng

Author for correspondence:

Department of Biomedical Engineering, University of Southern

California, Los Angeles, CA, USA and

Department of Electrical Engineering, University of Southern California, Los Angeles, CA, USA

ellis.meng@usc.edu

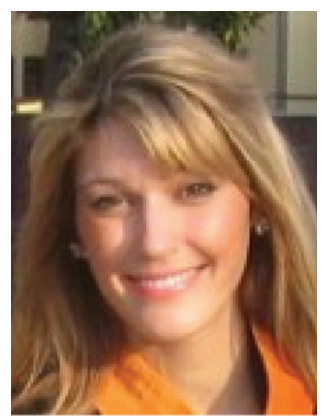

Roya Sheybani

Department of Biomedical Engineering, University of Southern

California, Los Angeles, CA, USA
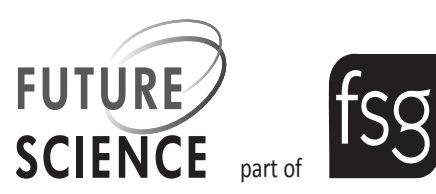
can be precisely controlled to avoid the peak and trough drug concentration profile in the plasma between successive doses and the corresponding pattern of action, leading to therapies that mimic the chronobiological pattern of the condition $[1,3]$.

In this article, three categories of drug-delivery systems utilizing micro- and nano-fabrication technologies are presented. An introduction to each category is provided followed by current research highlights. The article concludes with the authors' perspective on remaining challenges and future developments.

\section{Reservoir-based drug-delivery devices}

Microreservoirs temporarily store drug payloads until their desired release. In passive devices, drug is released slowly by osmotic or diffusive transport, or in response to an environmental stimulus. Alternatively, drug can be rapidly expelled at the desired time from a pre-pressurized reservoir or by using electrochemical, thermal or magnetic actuation. Drug reservoirs etched into silicon can be capped with removable metal membranes that can be selectively removed by electrochemical dissolution or electrothermal degradation to control initiation of release [4].

Passive devices are generally easier to fabricate and require no external power. However, these benefits may be outweighed by low release rates and slow response [6]. The delivery rate cannot be intentionally altered or the delivery be terminated once started. Such release may be susceptible to temperature, $\mathrm{pH}$, saccharide concentration and antigen concentration that fluctuate over the course of treatment [3]. The non-biodegradable osmotic Duros ${ }^{\circledR}$ pump developed by Durect Corporation was US FDA approved in 2000 for 1 year subcutaneous delivery of prostate cancer treatment at a constant rate. This product was discontinued and clinical trials for new indications suspended pending redesign of the delivery system to address performance issues caused by premature shutdown $[7,8]$. Durect Corporation is also developing an injectable biodegradable polymer containing lyophilized proteins and smallmolecule compounds [7]. The implantable MicroCHIPS $^{\circledR}$ device activates individually addressable reservoirs each holding $300 \mathrm{nl}$ of drug using a thermal mechanism that can be triggered wirelessly [8]. Dosing can be terminated without the need for device extraction. MicroCHIPS reported successful human clinical trials of subcutaneous delivery of an anabolic agent for the treatment of osteoporosis in 2012 [9].

\section{Drug infusion micropumps}

Drug infusion micropumps are either passive or active according to the mechanism used to control drug release. Passive mechanisms such as osmosis or the use of environmental stimulus responsive materials can supply the pressure differential necessary to pump drug. Although passive devices are less complex, they offer limited control over the delivery profile. Active pumps may use an electrical signal, radio frequency wave or magnetic wave to drive drug delivery. These therefore require power and are more complex but these tradeoffs may be offset by the precise metering and, in some cases, refill capabilities [3]. A comprehensive review of recent developments in micro infusion pumps and actuation mechanisms is in [4].

OmniPod, a wearable insulin pump, developed by Insulet Corp, allows for subcutaneous delivery via a small cannula. The drug payload in the disposable reservoir provides 72 hours of delivery. A shape memory alloy actuator controls pump activation and can be operated through a wireless handheld device [10]. The second generation of the device was approved by the FDA in 2013. Another wearable insulin drug-delivery system, containing a piezoelectric actuator, is being developed by Debiotech in Europe. The final stages of development entail further miniaturization to support complete implantation inside the body [11]. Replenish Inc is developing an implantable electrochemically driven, refillable ophthalmic micro-pump designed to allow nanoliter intraocular infusions to treat age-related macular degeneration and glaucoma [8].

\section{Nanoparticles \& silicon nanoporous}

\section{membranes}

Nanotechnology devices, such as nanovectors, nanoshells and nanoporous silicon membranes, are man-made structures characterized as having at least one dimension measuring $1-100 \mathrm{~nm}$. A range of drug-release-time profiles can be achieved by selecting the appropriate material system. Biodegradable porosified silicon provides rapid kinetics (minutes to hours) in contrast to biodegradable polymers (weeks to months). Metal-based nanoshells can be selectively activated through tissue irradiation with near-infrared light. Active recognition elements can also be added to the surface to attain molecular targeting. However, despite decades of research, the increased specificity at the expense of added complexity in nanoparticle preparation, increased particle size, risk of biological adverse reactions to the targeting agent and challenges in transport of particles to target sites all present significant obstacles to clinical translation with a few notable exceptions [12]. Abraxane ${ }^{\circledR}$, a $130 \mathrm{~nm}$ paclitaxel nanoparticle, developed by American Bioscience Inc., was approved by the FDA for the treatment of metastatic breast cancer and non-small cell lung cancer in 2005 [12]. In 2003, a US patent was awarded to iMEDD Inc., for nanoporous microparticles to treat systemically 
accessible solid tumors, specifically the multiple lesion sites associated with metastatic disease [13].

\section{Challenges \& future perspective}

Advances in implantable drug-delivery pumps promise to improve quality of life. To achieve optimized and personalized patient-tailored therapy, implantable pumps need to move beyond simple open-loop systems and obtain physiological feedback to confirm that drug release has resulted in the desired therapeutic effect. This can be achieved through the incorporation of physical sensors that provide information on pressure, flow rate or delivery volumes, as well as biosensors that can guide the therapeutic regimen based on the body's response to medication [2]. Proteus Digital Health developed an ingestible wireless sensor that monitors the identity and timing of pill ingestion in an effort to improve patient adherence to pharmaceutical therapy. In 2012, the sensor was approved for use with placebo pills [14]. An investigational implantable pump developed by Medallion Therapeutics, Inc. (St. Paul, MN, USA), includes integrated pressure sensors to track delivery [15]. Medtronic's FDA-approved artificial pancreas device system, although not implantable, combines glucose sensors with a wearable insulin pump. Sensor data can stop insulin delivery when glucose values reach a preset level [16].

\section{...new opportunities for drug-delivery technologies are being forged.}

Another emerging field is electroceuticals, in which electrical stimulation is employed to affect and modify functions of the body, sometimes in lieu of conventional drug therapy. Examples include the development of leadless pacemakers, modulation of gastric contractility to treat diabetes, and stimulation of the vagal nerve as a therapy for epilepsy and inflammatory diseases [17].

Recent innovations in digital and wireless health have enabled data communication with medical implants. Depending on the type of implant and its incorporated sensors, wireless communication may be used to monitor status and adjust device operation or achieve data transfer between the device and an internet-based network that could be shared with caregivers and healthcare providers [18]. However, including additional wireless electronics may increase device size, increase the drain rate of the battery and entail additional security measures [3].

The invasive nature of implantable drug-delivery systems and their potential role in supporting or sustaining human life or preventing impairment of human health, subjects these devices to highest level of scrutiny by the FDA. Sufficient evidence is required to prove safety and efficacy, necessitating non-clinical (related to biocompatibility, toxicology, immunology, stress, wear, etc.) as well as preclinical and clinical studies. Every stage of device development is undertaken with these regulatory endpoints in mind [19].

Early stage investment in medical devices has noticeably declined as a result of uncertainty in the regulatory approval environment for new devices, the focus of investors short-term pay offs and new legislation (the 2013 Medical Device Tax Act for sales of medical devices). For implants with substantial clinical need and market size, investments now occur late in the development process, typically after clinical validation and regulatory approvals [20]. Despite the changing medical device ecosystem, new opportunities for drug-delivery technologies are being forged. Large pharmaceutical companies are seeking strategic alliances with drugdelivery technology companies to enhance the performance of existing drugs and develop administration methods for novel pharmaceutical agents [1].

\section{Financial \& competing interests disclosure}

E Meng is the inventor (assignee) of patents on microfluidic devices, electrochemical micropumps, and sensors. She also has a significant financial interest in Fluid Synchrony LLC. The authors have no other relevant affiliations or financial involvement with any organization or entity with a financial interest in or financial conflict with the subject matter or materials discussed in the manuscript apart from those disclosed.

No writing assistance was utilized in the production of this manuscript.

\section{References}

1 Paolino D, Sinha P, Fresta M, Ferrari M. Drug delivery systems. In: Encyclopedia of Medical Devices and Instrumentation. Webster JG (Ed.), Wiley-Blackwell (2006).

2 Meng E, Hoang T. MEMS-enabled implantable drug infusion pumps for laboratory animal research, preclinical, and clinical applications. Adv. Drug Deliv. Rev. 64(14), 1628-1638 (2012).

3 Tng DJH, Hu R, Song P, Roy I, Yong KT. Approaches and challenges of engineering implantable microelectromechanical systems (MEMS) drug delivery systems for in vitro and in vivo applications. Micromachines 3(4), 615-631 (2012).

4 Meng E, Hoang T. Micro-and nano-fabricated implantable drug-delivery systems. Ther. Deliv. 3(12), 1457-1467 (2012).

5 Folkman J, Long DM. The use of silicone rubber as a carrier for prolonged drug therapy. J. Surg. Res. 4(3), 139-142 (1964).

6 Tsai N-C, Sue CY. Review of MEMS-based drug delivery and dosing systems. Sens. Actuators A Phys. 134(2), 555-564 (2007). 
7 Durect Corporation. Products (2014). www.durect.com/wt/durect/page_name/duros

8 Stevenson CL, Santini JT, Langer R. Reservoir-based drug delivery systems utilizing microtechnology. Adv. Drug Deliv Rev. 64(14), 1590-1602 (2012).

9 Farra R, Sheppard NF, Mccabe L et al. First-in-human testing of a wirelessly controlled drug delivery microchip. Sci. Transl. Med. 4(122), 122ra2 (2012).

10 Ochoa M, Mousoulis C, Ziaie B. Polymeric microdevices for transdermal and subcutaneous drug delivery. Adv. Drug Deliv. Rev. 64(14), 1603-1616 (2012).

11 Debiotech SA. Switzerland. The Nanopump: the Future of miniaturized drug delivery pumps. Nanopump (2014). www.debiotech.com

12 Ferrari M. Cancer nanotechnology: opportunities and challenges. Nat. Rev. Cancer 5(3), 161-171 (2005).

13 Martin FJ, Boiarski AA. Microfabricated nanopore device for sustained release of therapeutic agent, US20140079754 (2003).

14 Proteus Digital Health. Proteus Digital Health Announces FDA Clearance of Ingestible Sensor (2012).

www.proteus.com
15 Lawson EF, Wallace MS. Current developments in intraspinal agents for cancer and noncancer pain. Curr. Pain Headache Rep. 14(1), 8-16 (2010).

16 Sheldon A, Warren J. Medtronic Gains Approval of First Artificial Pancreas Device System with Threshold Suspend Automation (2013).

http://newsroom.medtronic.com

17 Sekhon BS. Bioelectronic medicine/electroceuticals - the emergence of new electronic. Int. J. Novel. Res. Eng. Pharm. Sci. 1(1), 58-63 (2014).

18 Panescu D. Wireless communication systems for implantable medical devices. IEEE Eng. Med. Biol. Mag. 27(2), 96-101 (2008).

19 Meng E, Sheybani R. Insight: implantable medical devices. Lab Chip 14(17), 3233-3240 (2014).

20 Thakor NV. Translating the brain-machine interface. Sci. Transl. Med. 5(210), 210-217 (2013). 